\title{
EXISTENCE AND UNIQUENESS OF SOLUTIONS OF A SEMILINEAR FUNCTIONAL-DIFFERENTIAL EVOLUTION NONLOCAL CAUCHY PROBLEM
}

\author{
KATARZYNA KOŁODZIEJ \\ Cracow University of Technology \\ Institute of Mathematics \\ Warszawska 24 \\ 31-155 Cracow, Poland
}

(Received December, 1998; Revised April, 1999)

\begin{abstract}
Two theorems about the existence and uniqueness of mild and classical solutions of a semilinear functional-differential evolution nonlocal Cauchy problem in a general Banach space are proved. Methods of semigroups and the Banach contraction theorem are applied.

Key words: Abstract Cauchy Problem, Evolution Equation, Functional-Differential Equation, Nonlocal Condition, Existence and Uniqueness of the Solutions, Mild and Classical Solutions, Banach Contraction Theorem.

AMS subject classifications: $34 \mathrm{G} 20,34 \mathrm{~K} 30,34 \mathrm{~K} 99,47 \mathrm{D} 03,47 \mathrm{H} 10$.
\end{abstract}

\section{Introduction}

In this paper we study the existence and uniqueness of mild and classical solutions of a semilinear functional-differential evolution nonlocal Cauchy problem in a general Banach space. Methods of $C_{0}$ semigroups and the Banach theorem about the fixed point are applied. The functional-differential evolution nonlocal Cauchy problem considered here is of the form

and

$$
\begin{gathered}
u^{\prime}(t)+A u(t)=F_{1}\left(t, u(t), u\left(\sigma_{1}(t)\right), \ldots, u\left(\sigma_{m}(t)\right)\right) \\
+\int_{t_{0}}^{t} F_{2}\left(t, s, u(s), \int_{t_{0}}^{s} f(s, \tau, u(\tau)) d \tau\right) d s, t \in\left(t_{0}, t_{0}+a\right]
\end{gathered}
$$

$$
u\left(t_{0}\right)+G(u)=u_{0},
$$

where $t_{0} \geq 0, a>0,-A$ is the infinitesimal generator of a $C_{0}$ semigroup of operators 
on a Banach space, $F_{i}(i=1,2), G, f, \sigma_{i}(i=1, \ldots, m)$ are given functions satisfying some assumptions and $u_{0}$ is an element of the Banach space.

The results obtained pertaining to the nonlocal evolution problem are generalizations of those given by Byszewski $[2,4,5]$, and by Balasubramaniam and Chandrasekaran [1]. Moreover, the results obtained concerning the evolution problem (1.1)(1.2), where $F_{2}=0$ and $G=0$, are generalizations of those given by Winiarska [10] and Pazy [9].

Nonlocal semilinear and nonlinear functional-differential evolution Cauchy problems in general Banach spaces have also been studied by Byszewski $[3,6,7]$ and by Lin, Liu [8].

\section{Notation and Definitions}

Let $E$ be a Banach space with norm $\|\cdot\|$ and let $\{T(t)\}_{t \geq 0}$ be a $C_{0}$ semigroup of operators on $E$.

In this paper we assume that $-A$ is the infinitesimal generator of a $C_{0}$ semigroup of operators on $E, D(A)$ is the domain of $A, t_{0} \geq 0, a>0$,

$$
\begin{gathered}
I:=\left[t_{0}, t_{0}+a\right], \quad \Delta:=\left\{(t, s): t_{0} \leq s \leq t \leq t_{0}+a\right\} \\
M:=\sup _{t \in[0, a]}\|T(t)\|_{B L(E, E)}, \\
X:=C(I, E)
\end{gathered}
$$

and

$$
\begin{gathered}
F_{1}: I \times E^{m+1} \rightarrow E, \quad F_{2}: \Delta \times E^{2} \rightarrow E, \quad G: X \rightarrow E \\
f: \Delta \times E \rightarrow E, \quad \sigma_{i}: I \rightarrow I \quad(i=1, \ldots, m)
\end{gathered}
$$

are given functions satisfying some assumptions.

In the sequel, the operator norm $\|\cdot\|_{B L(E, E)}$ will be denoted by $\|\cdot\|$.

We will need the following two definitions of mild and classical solutions of the nonlocal Cauchy problem (1.1)-(1.2):

Definition 2.1: A function $u \in X$ satisfying the integral equation

$$
\begin{gathered}
u(t)=T\left(t-t_{0}\right) u_{0}-T\left(t-t_{0}\right) G(u) \\
+\int_{t_{0}}^{t} T(t-s) F_{1}\left(s, u(s), u\left(\sigma_{1}(s)\right), \ldots, u\left(\sigma_{m}(s)\right)\right) d s \\
+\int_{t_{0}}^{t} T(t-s)\left(\int_{t_{0}}^{s} F_{2}\left(s, \tau, u(\tau), \int_{t_{0}}^{\tau} f(\tau, \mu, u(\mu)) d \mu\right) d \tau\right) d s, t \in I
\end{gathered}
$$

is said to be a mild solution of the nonlocal Cauchy problem (1.1)-(1.2) on $I$.

Definition 2.2: A function $u: I \rightarrow E$ is said to be a classical solution of the nonlocal Cauchy problem (1.1)-(1.2) on $I$ if: 
(i) $u$ is continuous on $I$ and continuously differentiable on $I \backslash\left\{t_{0}\right\}$,

(ii) $\quad u^{\prime}(t)+A u(t)=F_{1}\left(t, u(t), u\left(\sigma_{1}(t)\right), \ldots, u\left(\sigma_{m}(t)\right)\right)$

$$
+\int_{t_{0}}^{t} F_{2}\left(t, s, u(s), \int_{t_{0}}^{s} f(s, \tau, u(\tau)) d \tau\right) d s, \quad t \in I \backslash\left\{t_{0}\right\},
$$

(iii) $u\left(t_{0}+G(u)=u_{0}\right.$

\section{Theorem about a Mild Solution}

Theorem 3.1: Assume that

(i) for all $z_{i} \in E(i=0,1, \ldots, m)$, the function $I \ni t \rightarrow F_{1}\left(t, z_{0}, z_{1}, \ldots, z_{m}\right) \in E$ is continuous on $I$, for all $z_{i} \in E \quad(i=1,2)$ the function $\Delta \ni(t, s) \rightarrow$ $F_{2}\left(t, s, z_{1}, z_{2}\right) \in E$ is continuous on $\Delta$, for all $z \in E$ the function $\Delta \ni$ $(t, s) \rightarrow f(t, s, z) \in E$ is continuous on $\Delta, G: X \rightarrow E, \sigma_{i} \in C(I, I)(i=1, \ldots, m)$ and $u_{0} \in E$;

(ii) there are constants $L_{i}>0(i=1,2,3,4)$ such that

$$
\begin{gathered}
\left\|F_{1}\left(t, z_{0}, z_{1}, \ldots, z_{m}\right)-F_{1}\left(t, \tilde{z}_{0}, \tilde{z}_{1}, \ldots, \tilde{z}_{m}\right)\right\| \leq L_{1} \sum_{i=0}^{m}\left\|z_{i}-\tilde{z}_{i}\right\| \\
\text { for } t \in I, z_{i}, \tilde{z}_{i} \in E \quad(i=0,1, \ldots, m) \\
\left\|F_{2}\left(t, s, z_{1}, z_{2}\right)-F_{2}\left(t, s, \tilde{z}_{1}, \tilde{z}_{2}\right)\right\| \leq L_{2} \sum_{i=1}^{2}\left\|z_{i}-\tilde{z}_{i}\right\| \\
\qquad f o r(t, s) \in \Delta, z_{i}, \tilde{z}_{i} \in E \quad(i=1,2) ; \\
\|f(t, s, z)-f(t, s, \tilde{z})\| \leq L_{3}\|z-\tilde{z}\| \\
\text { for }(t, s) \in \Delta, z, \tilde{z} \in E \\
\|G(w)-G(\tilde{w})\| \leq L_{4}\|w-\tilde{w}\|_{X} \text { for } w, \widetilde{w} \in X
\end{gathered}
$$

(iii) $\quad M\left[L_{1} a(m+1)+L_{2} a^{2}\left(1+L_{3} a\right)+L_{4}\right]<1$.

Then the nonlocal Cauchy problem (1.1)-(1.2) has a unique mild solution on I.

Proof: Introduce an operator $\mathcal{F}$ on $X$ by the formula

$$
\begin{gathered}
(\mathscr{F} w)(t):=T\left(t-t_{0}\right) u_{0}-T\left(t-t_{0}\right) G(w) \\
+\int_{t_{0}}^{t} T(t-s) F_{1}\left(s, w(s), w\left(\sigma_{1}(s)\right), \ldots, w\left(\sigma_{m}(s)\right)\right) d s
\end{gathered}
$$




$$
+\int_{t_{0}}^{t} T(t-s)\left(\int_{t_{0}}^{s} F_{2}\left(s, \tau, w(\tau), \int_{t_{0}}^{\tau} f(\tau, \mu, w(\mu)) d \mu\right) d \tau\right) d s
$$

for $w \in X$ and $t \in I$.

It is easy to see that

$$
\mathscr{F}: X \rightarrow X
$$

Now, we shall show that $\mathscr{F}$ is a contraction on $X$. For this purpose, consider the difference

$$
\begin{gathered}
(\mathscr{F} w)(t)-(\mathscr{F} \widetilde{w})(t)=-T\left(t-t_{0}\right)[G(w)-G(\widetilde{w})] \\
+\int_{t_{0}}^{t} T(t-s)\left[F_{1}\left(s, w(s), w\left(\sigma_{1}(s)\right), \ldots, w\left(\sigma_{m}(s)\right)\right)\right. \\
\left.\quad-F_{1}\left(s, \widetilde{w}(s), \widetilde{w}\left(\sigma_{1}(s)\right), \ldots, \widetilde{w}\left(\sigma_{m}(s)\right)\right)\right] d s \\
+\int_{t_{0}}^{t} T(t-s)\left(\int _ { t _ { 0 } } ^ { s } \left[F_{2}\left(s, \tau, w(\tau), \int_{t_{0}}^{\tau} f(\tau, \mu, w(\mu)) d \mu\right)\right.\right. \\
\left.\left.\quad-F_{2}\left(s, \tau, \widetilde{w}(\tau), \int_{t_{0}}^{\tau} f(\tau, \mu, \tilde{w}(\mu)) d \mu\right)\right] d \tau\right) d s
\end{gathered}
$$

for $w, \widetilde{w} \in X$ and $t \in I$.

From (3.6), (2.1) and (3.1)-(3.4),

$$
\begin{gathered}
\|(\widetilde{F} w)(t)-(\widetilde{F} \tilde{w})(t)\| \leq\left\|T\left(t-t_{0}\right)\right\|\|G(w)-G(\widetilde{w})\| \\
+\int_{t_{0}}^{t}\|T(t-s)\| \| F_{1}\left(s, w(s), w\left(\sigma_{1}(s)\right), \ldots, w\left(\sigma_{m}(s)\right)\right) \\
\quad-F_{1}\left(s, \widetilde{w}(s), \widetilde{w}\left(\sigma_{1}(s)\right), \ldots, \widetilde{w}\left(\sigma_{m}(s)\right)\right) \| d s \\
+\int_{t_{0}}^{t}\|T(t-s)\|\left(\int_{t_{0}}^{s} \| F_{2}\left(s, \tau, w(\tau), \int_{t_{0}}^{\tau} f(\tau, \mu, w(\mu)) d \mu\right)\right) \\
\left.\quad-F_{2}\left(s, \tau, \widetilde{w}(t), \int_{t_{0}}^{\tau} f(\tau, \mu, \widetilde{w}(\mu)) d \mu\right) \| d \tau\right) d s \\
\leq M L_{4}\|w-\widetilde{w}\|_{X}+M L_{1} \int_{t_{0}}^{t}\left(\|w(s)-w(\widetilde{s})\|+\sum_{i=1}^{m}\left\|w\left(\sigma_{i}(s)\right)-\widetilde{w}\left(\sigma_{i}(s)\right)\right\|\right) d s
\end{gathered}
$$




$$
\begin{gathered}
+M L_{2} \int_{t_{0}}^{t}\left(\int_{t_{0}}^{s}\left[\|w(\tau)-\tilde{w}(\tau)\|+\int_{t_{0}}^{\tau}\|f(\tau, \mu, w(\mu))-f(\tau, \mu, \widetilde{w}(\mu))\| d \mu\right] d \tau\right) d s \\
\leq M L_{4}\|w-\tilde{w}\|_{X}+M L_{1} a(m+1)\|w-\tilde{w}\|_{X} \\
+M L_{2} \int_{t_{0}}^{t}\left(\int_{t_{0}}^{s}\left[\|w(\tau)-\tilde{w}(\tau)\|+L_{3} \int_{t_{0}}^{\tau}\|w(\mu)-\tilde{w}(\mu)\| d \mu\right] d \tau\right) d s \\
=M\left[L_{1} a(m+1)+L_{2} a^{2}\left(1+L_{3} a\right)+L_{4}\right]\|w-\widetilde{w}\|_{X}
\end{gathered}
$$

for $w, \widetilde{w} \in X$ and $t \in I$.

Let

$$
q:=M\left[L_{1} a(m+1)+L_{2} a^{2}\left(1+L_{3} a\right)+L_{4}\right]
$$

Then, by (3.7) and by assumption (iii),

$$
\|\mathscr{F} w-\mathscr{F} \widetilde{w}\|_{X} \leq q\|w-\widetilde{w}\|_{X} \text { for } w, \widetilde{w} \in X
$$

with $0<q<1$. This shows that operator $\mathscr{F}$ is a contraction on $X$.

Consequently, from (3.5) and (3.8), operator $\mathscr{F}$ satisfies all the assumptions of the Banach contraction theorem. Therefore, in space $X$ there is only one fixed point of $\mathcal{F}$ and this point is the mild solution of the nonlocal Cauchy problem (1.1)-(1.2). So the proof of Theorem 3.1 is complete.

\section{Theorem about a Classical Solution}

Theorem 4.1: Suppose that assumptions (i)-(iii) of Theorem 3.1 are satisfied. Then the nonlocal Cauchy problem (1.1)-(1.2) has a unique mild solution on I. Assume, additionally, that:

(i) $E$ is a reflexive Banach space, $u_{0} \in D(A)$ and $G(u) \in D(A)$, where $u$ denotes the unique mild solution of problem (1.1)-(1.2);

(ii) there are constants $C_{i}>0(i=1,2)$ such that

$$
\begin{gathered}
\left\|F_{1}\left(t, z_{0}, z_{1}, \ldots, z_{m}\right)-F_{1}\left(\tilde{t}, z_{0}, z_{1}, \ldots, z_{m}\right)\right\| \leq C_{1}|t-\tilde{t}| \\
\text { for } t, \tilde{t} \in I, z_{i} \in E(i=0,1, \ldots, m)
\end{gathered}
$$

and

$$
\begin{gathered}
\left\|F_{2}\left(t, s, z_{1}, z_{2}\right)-F_{2}\left(\tilde{t}, s, z_{1}, z_{2}\right)\right\| \leq C_{2}|t-\tilde{t}| \\
\quad \operatorname{for}(t, s) \in \Delta, \tilde{t}, s) \in \Delta, z_{i} \in E \quad(i=1,2)
\end{gathered}
$$


(iii) there is a constant $c>0$ such that

$$
\begin{gathered}
\left\|u\left(\sigma_{i}(t)\right)-u\left(\sigma_{i}(\tilde{t})\right)\right\| \leq c\|u(t)-u(\tilde{t})\| \\
\text { for } t, \tilde{t} \in I \quad(i=0,1, \ldots, m) .
\end{gathered}
$$

Then $u$ is the unique classical solution of the nonlocal Cauchy problem (1.1)-(1.2) on $I$.

Proof: Since all the assumptions of Theorem 3.1 are satisfied, then the nonlocal Cauchy problem (1.1)-(1.2) possesses a unique mild solution which, according to assumption $(i)$, is denoted by $u$.

Now, we shall show that $u$ is the unique classical solution of problem (1.1)-(1.2) on I. To this end, introduce

$$
N_{1}:=\max _{s \in I}\left\|F_{1}\left(s, u(s), u\left(\sigma_{1}(s)\right), \ldots, u\left(\sigma_{m}(s)\right)\right)\right\|
$$

and

$$
N_{2}: \underset{(\xi, \eta) \in \Delta}{=\max _{2}}\left\|F_{2}\left(\xi, \eta, u(\eta), \int_{t_{0}}^{\eta} f(\eta, \mu, u(\mu)) d \mu\right)\right\|
$$

and observe that

$$
\begin{gathered}
u(t+h)-u(t)=\left[T\left(t+h-t_{0}\right) u_{0}-T\left(t-t_{0}\right) u_{0}\right] \\
-\left[T\left(t+h-t_{0}\right) G(u)-T\left(t-t_{0}\right) G(u)\right] \\
+\int_{t_{0}}^{t_{0}+h} T(t+h-s) F_{1}\left(s, u(s), u\left(\sigma_{1}(s)\right), \ldots, u\left(\sigma_{m}(s)\right)\right) d s \\
+\int_{t_{0}+h}^{t+h} T(t+h-s) F_{1}\left(s, u(s), u\left(\sigma_{1}(s)\right), \ldots, u\left(\sigma_{m}(s)\right)\right) d s \\
-\int_{t_{0}}^{t} T(t-s) F_{1}\left(s, u(s), u\left(\sigma_{1}(s)\right), \ldots, u\left(\sigma_{m}(s)\right)\right) d s \\
+\int_{t_{0}}^{t_{0}+h} T(t+h-s)\left(\int_{t_{0}}^{s} F_{2}\left(s, \tau, u(\tau), \int_{t_{0}}^{\tau} f(\tau, \mu, u(\mu)) d \mu\right) d \tau\right) d s \\
+\int_{t_{0}+h}^{t+h} T(t+h-s)\left(\int_{t_{0}}^{s} F_{2}\left(s, \tau, u(\tau), \int_{t_{0}}^{\tau} f(\tau, \mu, u(\mu)) d \mu\right) d \tau\right) d s
\end{gathered}
$$




$$
\begin{aligned}
& -\int_{t_{0}}^{t} T(t-s)\left(\int_{t_{0}}^{s} F_{2}\left(s, \tau, u(\tau), \int_{t_{0}}^{\tau} f(\tau, \mu, u(\mu)) d \mu\right) d \tau\right) d s \\
& =T\left(t-t_{0}\right)[T(h)-I] u_{0}-T\left(t-t_{0}\right)[T(h)-I] G(u) \\
& +\int_{t_{0}}^{t_{0}+h} T(t+h-s) F_{1}\left(s, u(s), u\left(\sigma_{1}(s)\right), \ldots, u\left(\sigma_{m}(s)\right)\right) d s \\
& +\int_{t_{0}}^{t} T(t-s)\left[F_{1}\left(s+h, u(s+h), u\left(\sigma_{1}(s+h)\right), \ldots, u\left(\sigma_{m}(s+h)\right)\right)\right. \\
& \left.-F_{1}\left(s, u(s), u\left(\sigma_{1}(s)\right), \ldots, u\left(\sigma_{m}(s)\right)\right)\right] d s \\
& +\int_{t_{0}}^{t_{0}+h} T(t+h-s)\left(\int_{t_{0}}^{s} F_{2}\left(s, \tau, u(\tau), \int_{t_{0}}^{\tau} f(\tau, \mu, u(\mu)) d \mu\right) d \tau\right) d s \\
& +\int_{t_{0}}^{t} T(t-s)\left(\int _ { t _ { 0 } } ^ { s } \left[F_{2}\left(s+h, \tau, u(\tau), \int_{t_{0}}^{\tau} f(\tau, \mu, u(\mu)) d \mu\right)\right.\right. \\
& \left.\left.-F_{2}\left(s, \tau, u(\tau), \int_{t_{0}}^{\tau} f(\tau, \mu, u(\mu)) d \mu\right)\right] d \tau\right) d s \\
& +\int_{t_{0}}^{t} T(t-s)\left(\int_{s}^{s+h} F_{2}\left(s+h, \tau, u(\tau), \int_{t_{0}}^{\tau} f(\tau, \mu, u(\mu)) d \mu\right) d \tau\right) d s
\end{aligned}
$$

for $t \in\left[t_{0}, t_{0}+a\right), h>0$ and $t+h \in\left(t_{0}, t_{0}+a\right]$.

Consequently, by (4.6), (2.1) and (4.1)-(4.5),

$$
\begin{aligned}
& \|u(t+h)-u(t)\| \leq h M\left\|A u_{0}\right\|+h M\|A G(u)\|+h M N_{1}+a h M L_{1} \\
& +M L_{1} \int_{t_{0}}^{t}\left(\|u(s+h)-u(s)\|+\sum_{i=1}^{m}\left\|u\left(\sigma_{i}(s+h)\right)-u\left(\sigma_{i}(s)\right)\right\|\right) d s \\
& +a^{2} M L_{2} h+2 a M N_{2} h \leq C h+M L_{1}(1+m c) \int_{t_{0}}^{t}\|u(s+h)-u(s)\| d s
\end{aligned}
$$

for $t \in\left[t_{0}, t_{0}+a\right), h>0$ and $t+h \in\left(t_{0}, t_{0}+a\right]$, where 


$$
C:=M\left[\left\|A u_{0}\right\|+\|A G(u)\|+N_{1}+a L_{1}+a^{2} L_{2}+2 a N_{2}\right]
$$

From (4.7) and Gronwall's inequality,

$$
\|u(t+h)-u(t)\| \leq C e^{a M L_{1}(1+m c)} h
$$

for $t \in\left[t_{0}, t_{0}+a\right), h>0$ and $t+h \in\left(t_{0}, t_{0}+a\right]$. Hence $u$ is Lipschitz continuous on $I$.

The Lipschitz continuity of $u$ on $I$ and inequalities (4.1), (3.1), (4.2) imply that the function

$$
\begin{gathered}
I \ni t \rightarrow k(t):=F_{1}\left(t, u(t), u\left(\sigma_{1}(t)\right), \ldots, u\left(\sigma_{m}(t)\right)\right) \\
+\int_{t_{0}}^{t} F_{2}\left(t, s, u(s), \int_{t_{0}}^{s} f(s, \tau, u(\tau)) d \tau\right) d s \in E
\end{gathered}
$$

is Lipschitz continuous on $I$. This property of $t \rightarrow k(t)$ together with assumptions of Theorem 4.1 imply by Theorem 1 from [10], by Theorem 3.1 from this paper and by (2.2), that the linear Cauchy problem

$$
\begin{aligned}
v^{\prime}(t)+A v(t) & =k(t), t \in I \backslash\left\{t_{0}\right\}, \\
v\left(t_{0}\right) & =u_{0}-G(u)
\end{aligned}
$$

has a unique classical solution $v$ such that

$$
\begin{gathered}
v(t)=T\left(t-t_{0}\right) u_{0}-T\left(t-t_{0}\right) G(u)+\int_{t_{0}}^{t} T(t-s) k(s) d s \\
=T\left(t-t_{0}\right) u_{0}-T\left(t-t_{0}\right) G(u) \\
+\int_{t_{0}}^{t} T(t-s) F_{1}\left(s, u(s), u\left(\sigma_{1}(s)\right), \ldots, u\left(\sigma_{m}(s)\right)\right) d s \\
+\int_{t_{0}}^{t} T(t-s)\left(\int_{t_{0}}^{s} F_{2}\left(s, \tau, u(\tau), \int_{t_{0}}^{\tau} f(\tau, \mu, u(\mu)) d \mu\right) d \tau\right) d s=u(t), t \in I .
\end{gathered}
$$

Consequently, $u$ is the unique classical solution of the nonlocal Cauchy problem (1.1)(1.2) on $I$. Therefore, the proof of Theorem 4.1 is complete.

\section{References}

[1] Balasubramaniam, P. and Chandrasekaran, M., Existence of solutions of nonlinear integrodifferential equation with nonlocal boundary conditions in Banach space, Atti Sem. Mat. Fis. Univ. Modena 46 (1998), 1-13. 
[2] Byszewski, L., Theorems about the existence and uniqueness of solutions of a semilinear evolution nonlocal Cauchy problem, J. Math. Anal. Appl. 162:2 (1991), 494-505.

[3] Byszewski, L., Uniqueness criterion for solution of abstract nonlocal Cauchy problem, J. Appl. Math. Stoch. Anal. 6 (1993), 49-54.

[4] Byszewski, L., Differential and Functional-Differential Problems with Nonlocal Conditions, Cracow University of Technology, Monograph 184, Cracow 1995.

[5] Byszewski, L., Existence and uniqueness of mild and classical solutions of semilinear functional-differential evolution nonlocal Cauchy problem, Selected Probl. in Math., Cracow Univ. of Tech., Anniversary Issue 6 (1995), 25-33.

[6] Byszewski, L., On weak solutions of functional-differential abstract nonlocal Cauchy problem, Annales Polonici Math. 65 (1997), 163-170.

[7] Byszewski, L., Application of properties of the right-hand sides of evolution equations to an investigation of nonlocal evolution problems, Nonlinear Anal. 33 (1998), 413-426.

[8] Lin, Y. and Liu, J.H., Semilinear integrodifferential equations with nonlocal Cauchy problem, Nonlinear Anal. 26 (1996), 1023-1033.

[9] Pazy, A., Semigroups of Linear Operators and Applications to Partial Differential Equations, Springer-Verlag, New York, Berlin, Tokyo 1983.

[10] Winiarska, T., Nonlinear evolution equation with a parameter, Bull. Polon. Acad. Sci. Math Ser. 37 (1989), 157-162. 


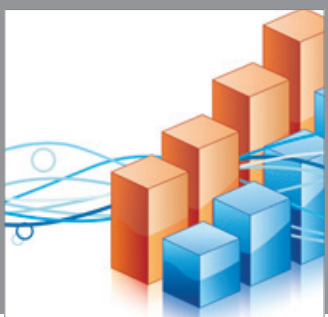

Advances in

Operations Research

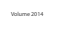

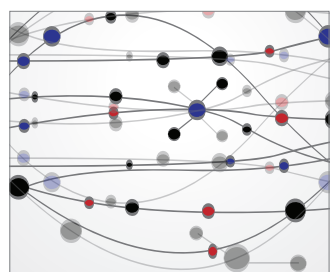

\section{The Scientific} World Journal
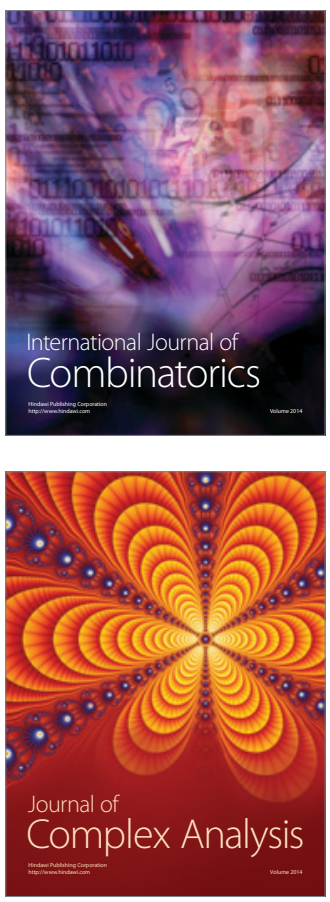

International Journal of

Mathematics and

Mathematical

Sciences
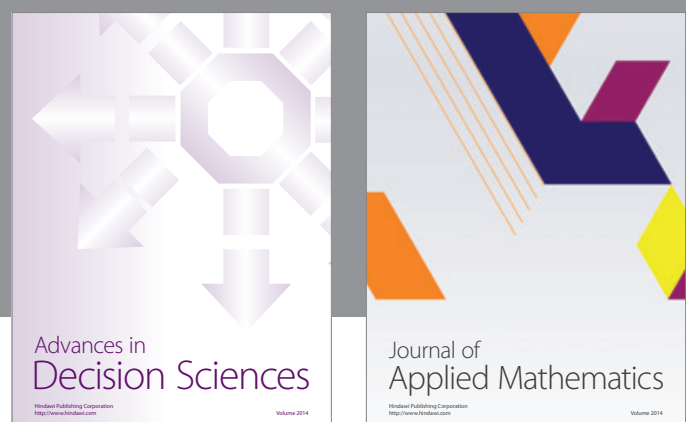

Journal of

Applied Mathematics
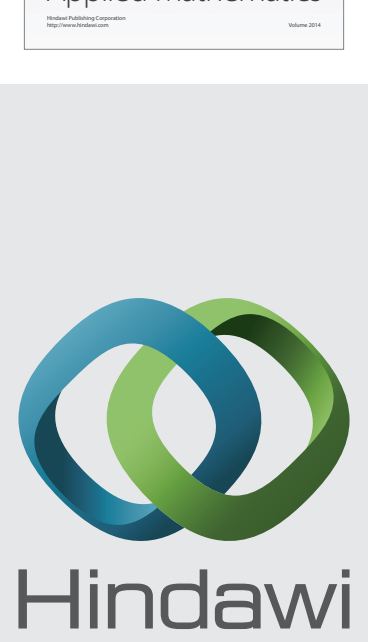

Submit your manuscripts at http://www.hindawi.com
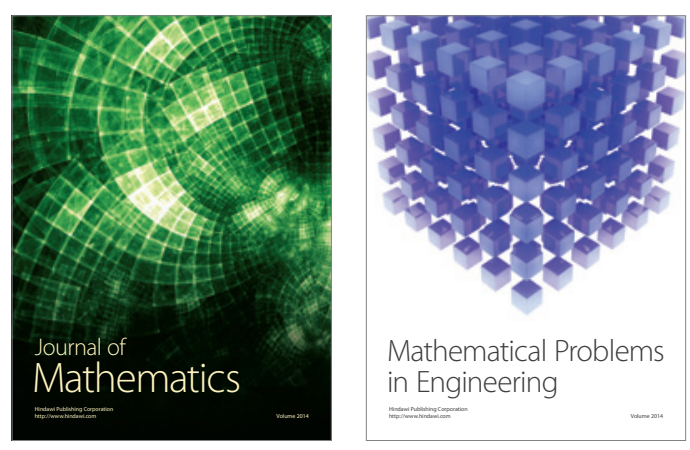

Mathematical Problems in Engineering
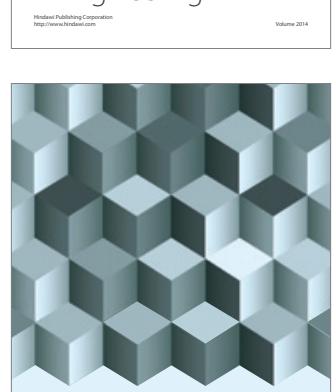

Journal of

Function Spaces
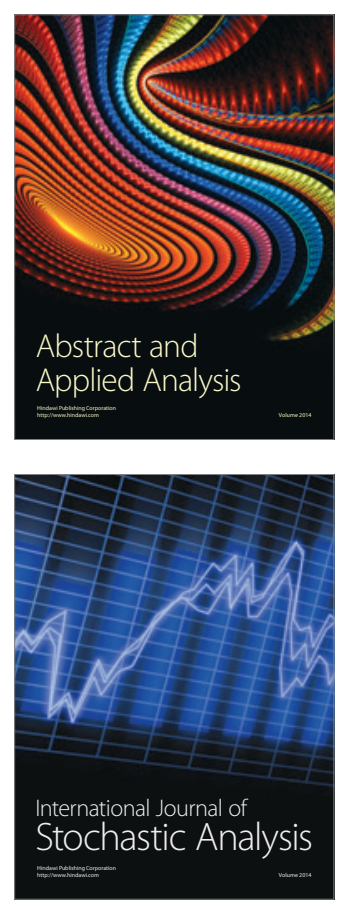

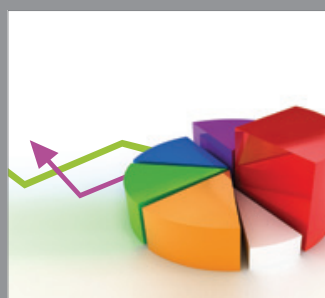

ournal of

Probability and Statistics

Promensencen
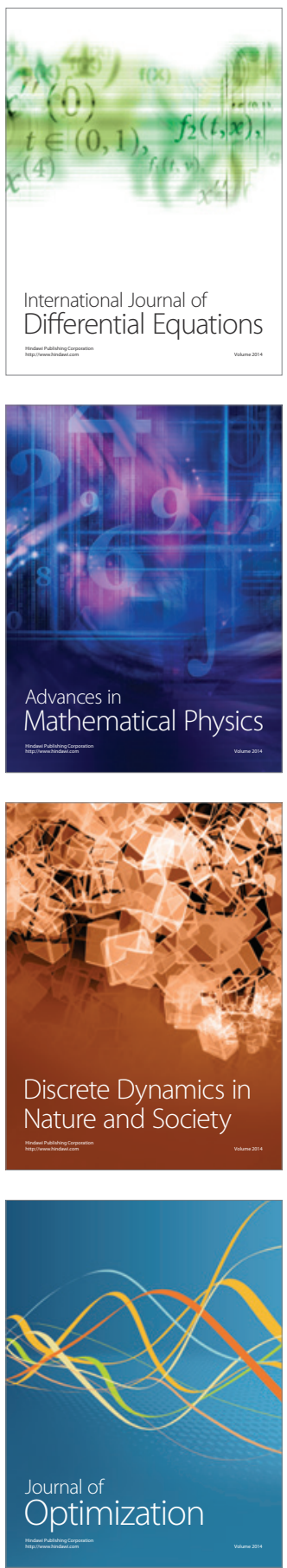